\title{
The gerC locus of Bacillus subtilis, required for menaquinone biosynthesis, is concerned only indirectly with spore germination
}

Krebs Institute for Biomolecular Research, Dept of Molecular Biology and Biotechnology, University of Sheffield, Sheffield S10 2TN, UK

\author{
A. J. Howard Leatherbarrow, Mohammed A. Yazdi, Janet P. Curson \\ and Anne Moir
}

Author for correspondence: Anne Moir. Tel: +44 1142224418. Fax: +44 1142728697.
e-mail: a.moir@sheffield.ac.uk

\begin{abstract}
The gerC region of Bacillus subtilis comprises a tricistronic operon, encoding enzymes that catalyse the late stages of menaquinone biosynthesis. The gerC58 mutation is responsible for a severe growth defect; unsuppressed mutant cells grow as very short rods, which sometimes septate aberrantly. Cultures grow only to a low cell density, rapidly lose viability, and never sporulate. Unlinked suppressor mutations can restore near-normal growth. Several independent suppressed isolates were examined; all grew to normal cell length, but they showed, to varying extents, a residual defect in the placement of the cell division septum. The germination properties of the suppressed derivatives varied from normal to significantly slow in germination in all germinants; therefore, the combination of the gerC mutation and different suppressor alleles resulted in spores with very different germination properties. This suggests that any relationship between the gerc gene products and spore germination is indirect. The gerCC58 mutation maps in a gene encoding the catalytic subunit of the heptaprenyldiphosphate synthase, which is responsible for formation of the isoprenoid side chain of menaquinone-7, and it is proposed that the gerCA, gerCB and gercC genes be renamed hepA, menG and hepB, respectively.
\end{abstract}

Keywords: spore germination, Bacillus subtilis, menaquinone biosynthesis, hep genes, men $G$

\section{INTRODUCTION}

The gerC mutants of Bacillus subtilis initially characterized by Trowsdale \& Smith (1975) were reported as temperature sensitive in spore germination in response to alanine, but normal in germination in the alternative combination of asparagine, glucose, fructose and $\mathrm{KCl}$. The gerC locus was mapped, by PBS1 transduction, to the lys-trp region of the chromosome, at $204^{\circ}$, but the original mutant strains contained several complicating linked mutations (Moir et al., 1979).

The gerC58 mutation affects vegetative cell growth, as was revealed when the gerC58 mutation was introduced into an unmutagenized background by transformation. The resulting gerC58 transformants formed very small

The GenBank accession number for the sequence reported in this paper is M80245. colonies on minimal medium and failed to grow at all on rich medium (the 'tiny' or Tny ${ }^{-}$phenotype; Yazdi $\&$ Moir, 1990). The original germination-defective isolate therefore carried, in addition to gerC58, an extragenic suppressor of gerC58, named sup-58, which restored approximately normal growth and spore formation. The ger $C$ locus was cloned in a B. subtilis $\phi 105$ vector, on a $6 \mathrm{~kb} \mathrm{Bcll}$ fragment of chromosomal DNA ( $\phi \mathrm{MY} 1$; Yazdi \& Moir, 1990), and the sequence was deposited in GenBank. The ger $C A$ and ger $C C$ genes encode the two subunits of heptaprenyl-diphosphate synthase, which catalyses the synthesis of the isoprenoid side chain of menaquinone-7, the predominant form found in bacilli (Zhang et al., 1997), and the gerCB gene encodes a methyltransferase responsible for the methylation of demethylmenaquinone (Koike-Takeshita et al., 1997). This paper examines the growth and germination defects in gerC mutant and suppressed strains, to determine whether this locus has a specific role in alanine germination. 


\section{METHODS}

Bacterial strains and media. Escherichia coli strains used for routine transformation with plasmids and M13 clones were DH $5 \alpha$ and JM101, respectively. B. subtilis strains are listed in Table 1, and are described elsewhere in the text. These were routinely grown and stored on nutrient agar (Oxoid), except where stated. Spores were prepared on the same medium, and washed as described previously (Moir et al., 1979). Germination was monitored as fall in $\mathrm{OD}_{580}$ after addition of germinants, by the procedure described by Yazdi \& Moir (1990). Minimal glucose medium was that of Anagnostopoulos \& Spizizen (1961), supplemented with any amino acid requirements. Transformation of $E$. coli was by the method of Mandel \& Higa (1970).

Plasmids and phages. A $B c l I-S s t I$ segment, which includes the entire gerC-complementing region, was excised from phage MY1 as an SstI fragment (using the vector SstI site adjacent to the BclI site) and subcloned into pUC119 to yield plasmid pMAY1 (Yazdi, 1989). The approximately 700 bp PvuII-SstI fragment in M13mp18 clone PS2 (Fig. 1), carrying the end of
gerCC and the start of $n d k$, was excised as a $B a m \mathrm{HI}-E c o \mathrm{RI}$ fragment using flanking M13 cloning sites and cloned into the integrational vector pAZ106 (Zuberi et al., 1990) to yield plasmid pHL1. Plasmid pHL2 carries the approximately 400 bp PstI-PvuII fragment internal to gerCC (fragment 2 in Fig. 1), excised from an M13 clone (using flanking sites) as a $S p h I-E c o R I$ fragment and cloned into the equivalent sites in the polylinker of pAZ106.

Transformation of competent B. subtilis cells. The cells were transformed as described by Warburg \& Moir (1981). Recombinants were purified by single colony isolation before testing of unselected markers.

Spore germination assays. The tetrazolium reaction used to score $\mathrm{Ger}^{+}$and $\mathrm{Ger}^{-}$colonies as Tzm-Red or Tzm-White, respectively, was as described previously by Zuberi et al. (1985). For assay of germination rates, spores were prepared on nutrient agar and washed as described by Moir et al. (1979). Germination was monitored as fall in $\mathrm{OD}_{580}$ after addition of germinants, by the procedure described in Moir $e t$ al. (1979), except that Tris/ $\mathrm{HCl}$ was $10 \mathrm{mM}, \mathrm{pH} 8 \cdot 4$, and $\mathrm{KCl}$

\section{Table 1. Strains of $B$. subtilis used}

\begin{tabular}{|c|c|c|}
\hline Strain & Genotype & Source/reference \\
\hline BD111 & thrA5 cysB3 $\operatorname{trp} C 2$ & D. Dubnau (Public Health Institute, New York) \\
\hline 1604 & $\operatorname{trpC2}$ & Moir et al. (1979); PBS1 transduction × BD111 \\
\hline 1558 & gerC58 wrd tzm(gerJ51) trpC2 & Moir et al. (1979) \\
\hline AM677 & thrA5 trpC2 gerC58 sup-58 & Yazdi \& Moir $(1990)$; DNA $(1558) \times$ BD111, by congression \\
\hline AM1136 & $\operatorname{trpC2} \sup -58$ & $\begin{array}{l}\text { DNA }(1604) \times \text { AM677, selecting } \mathrm{Thr}^{+} ; \text {ger } \mathrm{C}^{+} \text {introduced by } \\
\text { congression; presence of gerC }{ }^{+} \text {and sup- } 58 \text { each proved by } \\
\text { transformation linkage in back-crosses (Leatherbarrow, 1994) }\end{array}$ \\
\hline AM1228 & $\operatorname{trpC2}$ leuB8 gerC58 & DNA (AM677) $\times$ QB99 by transformation; unstable \\
\hline QB99 & bisH2 leuB8 & Bacillus Genetic Stock Centre IA89 \\
\hline S3, S5, S7 & $\operatorname{trp} C 2$ leuB8 gerC58, suppressed & Independent derivatives of AM1228 \\
\hline
\end{tabular}
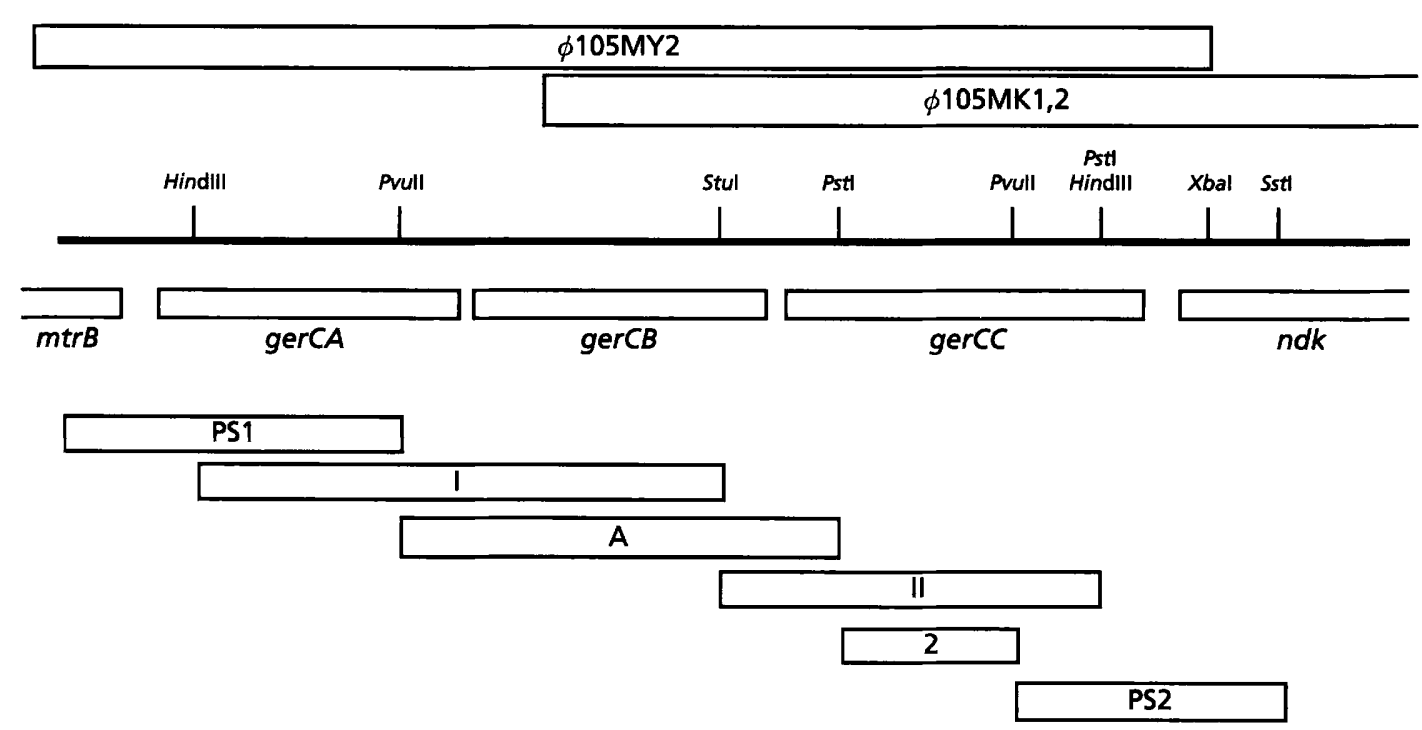

Fig. 1. gerC region of the $B$. subtilis chromosome. The positions of ger $C$ and flanking genes are indicated, as well as the relative locations of the cloned fragments in the phage and plasmid subclones. The inserts in integrational plasmids pHL1 and $\mathrm{pHL} 2$ are fragments 'PS2' and ' 2 ', respectively. 
was $2.25 \mathrm{mg} \mathrm{m}^{-1}$. Germination rates were calculated over $90 \mathrm{~min}$ from addition of germinant.

Subcloning and sequencing techniques. Standard DNA manipulation procedures (Sambrook et al., 1989) were used. Sequencing of M13 single-stranded and plasmid doublestranded templates was by the dideoxy chain-termination procedure (Sanger et al., 1977). The DNA was radiolabelled using $\left[{ }^{35} \mathrm{~S}\right] \mathrm{dATP} \alpha \mathrm{S}$ (Amersham) and Sequenase (US Biochemical). Sequences were determined on both strands and all restriction sites were fully overlapped. Sequences were assembled and analysed using the ANALYSEQ suite of programs (Staden, 1990).

Electron microscopy. Samples of vegetative cells were harvested and then washed twice in Spizizen salts. The pellet was resuspended in $50 \mathrm{ml}$ formaldehyde/glutaraldehyde fixative (Karnovsky, 1966) and incubated at room temperature for $2 \mathrm{~h}$. The samples were then washed five times in $0.1 \mathrm{M}$ sodium cacodylate, then post-fixed in $2 \%$ osmium tetroxide. After dehydration in an ethanol series, the material was incubated with dried absolute alcohol, then with propylene oxide, and incubated overnight in a 1:1 mixture of propylene oxide and araldite. Two $3 \mathrm{~h}$ incubations in fresh $100 \%$ araldite were followed by embedding in $100 \%$ araldite and polymerization at $60^{\circ} \mathrm{C}$ for $2 \mathrm{~h}$. Sections were cut, stained in saturated uranyl acetate, then washed and stained in Reynolds lead citrate solution. After a final wash, samples were examined under a Philips 301 transmission electron microscope.

\section{RESULTS AND DISCUSSION}

\section{Nucleotide sequence and functions of the gerC region}

Phage $\phi 105 \mathrm{MY} 2$, which retains a $3 \mathrm{~kb} \mathrm{BclI-Xbal} \mathrm{frag-}$ ment of the cloned DNA, still complements the growth defect of a gerC mutant, and thus carries a functional copy of the gerC locus (Yazdi \& Moir, 1990; Fig. 1) The $B c l \mathrm{I}-S s t \mathrm{I}$ sequence extending across the gerC region was subcloned from $\mathrm{PMAY} 1$ into M13 vectors and sequenced from defined restriction sites, using oligonucleotide primers to extend the sequence where necessary. The overall gene organization deduced from this DNA sequence is shown in Fig. 1.

The ger $C$ locus contains a cluster of three complete ORFs, designated gerCA, ger $C B$ and gerCC, of 251, 263 and 320 codons, respectively. These lie on the trpproximal side of the $m t r B$ gene (Gollnick et al., 1990), separated from $m t r B$ by a potential factor-independent terminator sequence in a $262 \mathrm{bp}$ intergenic region. The close coupling of the three gerC ORFs suggests that they form an operon, although no experimental assessment of the transcriptional unit has been made. Downstream of the gerC genes, an intergenic space of $115 \mathrm{bp}$ is followed by a homologue of the nucleoside diphosphate kinase ( $n d k)$ genes of various prokaryote and eukaryote species. There is no potential stem-loop structure that might serve as a factor-independent transcriptional terminator in the gerCC-ndk intergenic region.

The GerCA and GerCC proteins are $36 \%$ and $65 \%$ identical, respectively, to the two protein subunits of heptaprenyl-diphosphate synthase of Bacillus stearo- thermophilus ATCC 10149 (Koike-Takeshita et al., 1995), involved in the biosynthesis of the isoprenoid side chain of menaquinone-7. Zhang et al. (1997) have overexpressed the PCR-amplified ger CA and gerCC genes of $B$. subtilis, and demonstrated directly that they encode proteins with the same activity as their $B$. stearothermophilus homologues. orf- 1 and orf-3 are encoded as the first and third genes in a tricistronic operon, and flank an ORF of similar size to GerCB: the sequence of orf- 2 has recently been reported, and the gene demonstrated to encode a 2-heptaprenyl-1,4-naphthoquinone methyltransferase, $75 \%$ identical in amino acid sequence to GerCB (Koike-Takeshita et al., 1997).

\section{The phenotype of gerC mutant strains}

Unsuppressed gerC mutants can be generated in a genetic cross but are barely viable, and can only be maintained for up to two or three subcultures on minimal agar, as required for purification of recombinants, before suppressed derivatives outgrow the very slow growing mutant strain. Attempts to grow the mutant in rich medium fail, and result in even more rapid selection of suppressed derivatives (Yazdi \& Moir, 1990). In an attempt to clarify the relationship between the gerC58 allele (and its suppressors) and germination, additional suppressed mutants were isolated. Cultures of AM1228, each grown from a single colony in minimal medium to an $\mathrm{OD}_{600}$ of 0.2 , were concentrated 10 -fold and $0.1 \mathrm{ml}$ was plated on nutrient agar. Colonies of suppressed derivatives (around 50 per plate) appeared after $2 \mathrm{~d}$ incubation. Strains S3, S5 and S7 represent the most fully characterized independent isolates. Their spore germination properties were compared with 1604 $\left(\mathrm{gerC}^{+}\right)$, with AM677 (gerC58 sup-58) and with AM1136, a $\operatorname{ger}^{+}$sup-58 recombinant. The retention of the gerC58 mutation in suppressed strains could be checked in back-crosses, as already described for AM677 (Yazdi \& Moir, 1990). In the tetrazolium plate assay, 1604 and AM1136 were Tzm-Red, whereas all the gerC58-carrying strains (AM677 and all the new suppressed derivatives) were Tzm-White.

The original suppressed mutant, AM677, was temperature sensitive for alanine germination - the phenotype that gave rise to the gerC nomenclature. Spore germination of AM677 was $80-100 \%$ of the wild-type rate in a combination of asparagine, glucose, fructose and $\mathrm{KCl}$ at $42{ }^{\circ} \mathrm{C}$, and in both types of germinant at $25^{\circ} \mathrm{C}$, but was $<5 \%$ of the wild-type rate in alanine at $42{ }^{\circ} \mathrm{C}$. Spores of the sup-58 gerC $^{+}$mutant (AM1136), although apparently $\mathrm{Ger}^{+}$on a tetrazolium plate test, were rather slower to germinate than wild-type in both germinants at both temperatures $(50-70 \%$ of the wildtype rate). The germination properties of new isolates, all carrying both gerC58 and a suppressing mutation, varied. Spores of isolate S3 were somewhat slow to germinate in both germinants (rates similar to those of AM1136), whereas spores of S5 and S7 were approximately normal in germination $(80-110 \%$ of wild-type rates). None of the three showed any temperature- 


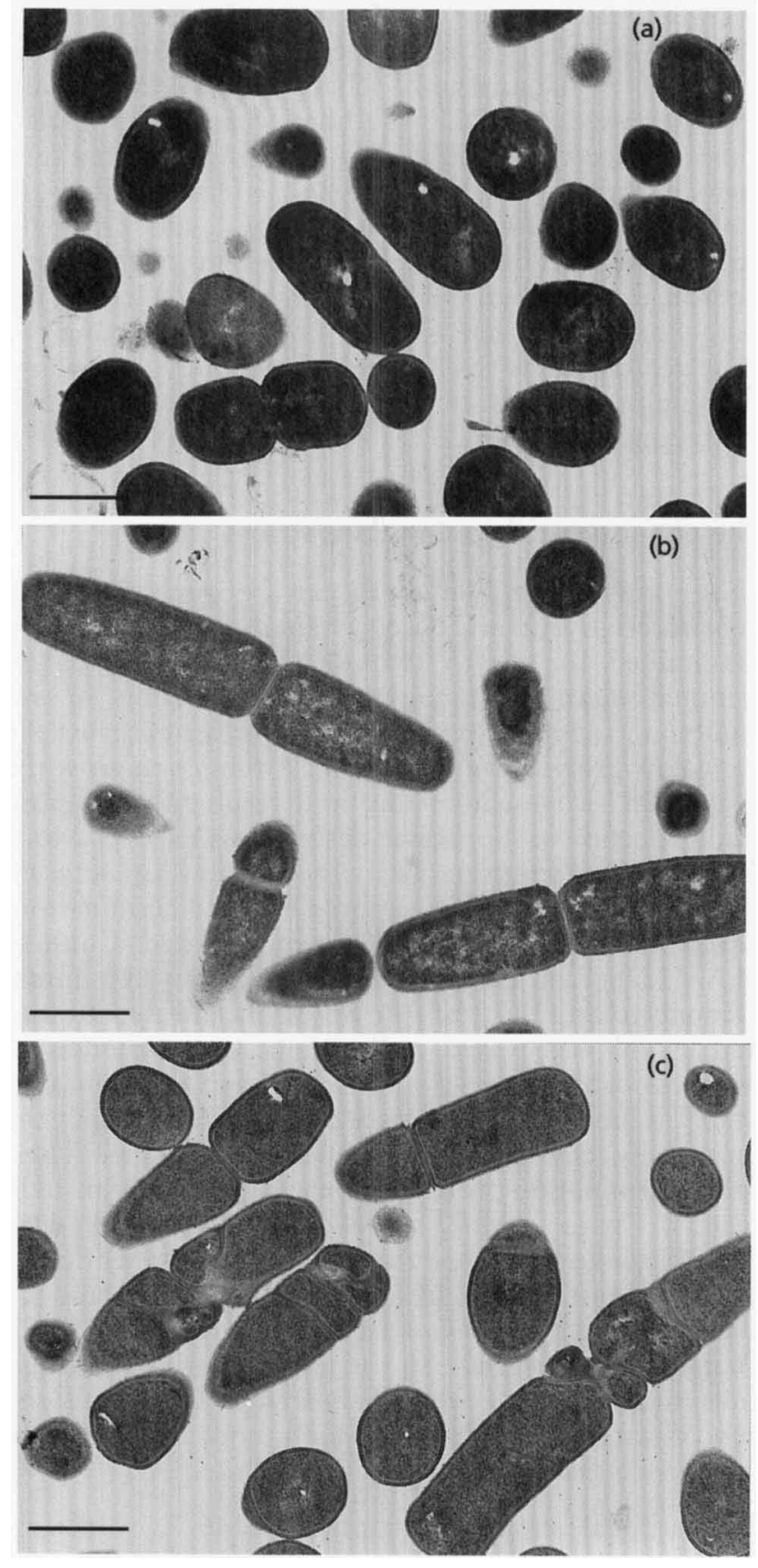

Fig. 2. Electron micrographs of thin sections of (a) a gerC58 unsuppressed strain, (b) wild-type strain 1604 and (c) gerC58 sup-58 strain AM677, all at the same magnification. Bar, $1 \mu \mathrm{m}$.

dependent effects. This variability implies that the germination behaviour is not defined solely by the presence of the gerC58 allele, but is highly dependent on the combination of gerC58 and suppressor alleles, and therefore on the nature or degree of suppression. Germination in alanine and/or in the asparagine, glucose, fructose and $\mathrm{KCl}$ combination can be affected - the first suppressed strain to be identified, AM677, is not typical. It is therefore unlikely that the gerC58 mutation lies in a gene with a direct role in alanine
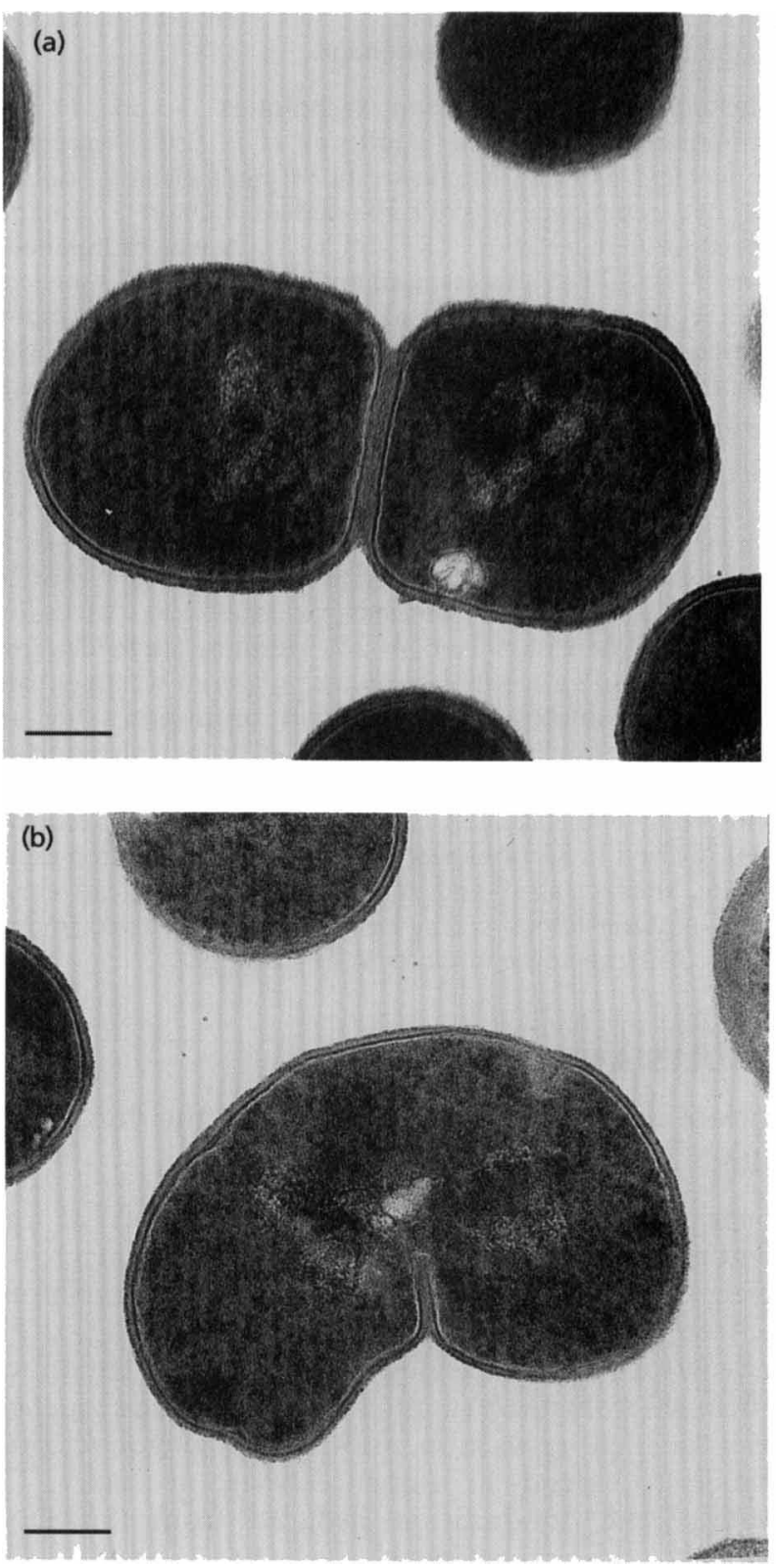

Fig. 3. Electron micrographs of thin sections of gerC58 unsuppressed mutant cells. (a) Normal transverse septum formation; (b) abnormal septation. Bar, $0.25 \mu \mathrm{m}$.

germination, as had been suggested by Moir et al. (1991).

\section{Morphology of gerC mutant cells}

Cultures of strains were grown in minimal medium, and harvested in mid-exponential growth $\left(\mathrm{OD}_{600}=0.1\right.$ for AM1228 and $0.3-0.5$ for all other strains). Vegetative cells of the unsuppressed gerC58 mutant (AM1228) are morphologically abnormal. Electron microscopy of thin sections has revealed that the cells are unusually short, but of approximately normal diameter (Figs $2 \mathrm{a}$ and $3 \mathrm{a}$ ); although most division events appear normal, approxi- 
mately $15 \%$ of the dividing cells have septal abnormalities, as shown in Fig. 3(b).

Although the suppressed mutant AM667 (Yazdi \& Moir, 1990) grows at an approximately normal rate in minimal and rich media (data not shown), at least $30 \%$ of the cells in the culture have an abnormality in cell division (Fig. 2c), possessing multiple, mislocated septa. Similar septal defects were also observed in cultures of the other suppressed mutants S3, S5 and S7, but were an order of magnitude less frequent in the population than was the case for AM677.

The defect in AM677 does not affect the localization or development of the sporulation septum, and the morphology of cross-sections of mature spores of AM677 and of the other suppressed mutants also appeared normal (data not shown). A derivative of AM677 which is $\operatorname{gerC}^{+}$but retains the sup-58 mutation (AM1136) has no defect in septum localization.

\section{Localization of the gerC58 mutation to the gerCC gene}

Phage $\phi 105 \mathrm{MY} 2$, a $2.9 \mathrm{~kb}$ deletion derivative of the original gerC clone, repairs the unsuppressed gerC58 mutant phenotype efficiently, by complementation, when it lysogenizes the strain (Yazdi \& Moir, 1990). Phage $\phi 105 M K 1,2$ (M. Kuroda \& C. Yanofsky, unpublished) contains DNA that partially overlaps this insert (Fig. 1). Infection of the unsuppressed gerC mutant strain by MK1,2 phage results in the formation of normally growing colonies at a low frequency, indicating that the repair is by recombination instead of complementation. The gerC58 mutation therefore lies within the second half of gerCB or in gerCC (although the latter gene is intact in $\phi 105 \mathrm{MK} 1,2$, it would be promoterless).

Regions of DNA (I, II, A and PS2; Fig. 1) had been subcloned into M13 as part of the sequencing programme. Replicative form DNAs from these subclones were used in transformation experiments to locate the gerC58 mutation. Strain AM677 (thrA5 trpC2 gerC58 sup-58) was transformed to $\mathrm{Thr}^{+}$by a limiting quantity $(0.01 \mu \mathrm{g})$ of chromosomal DNA from $1558(\operatorname{trpC2}$ gerC58 sup-58), in the presence of $10 \mu \mathrm{g}$ of the subclone DNA. Congression occurs at high DNA concentrations, so that a proportion of the $\mathrm{Thr}^{+}$transformants would be also transformed by the added gerC-derived DNA. Only the PS2 clone could provide gerC $^{+}\left(17\right.$ out of $384 \mathrm{Thr}^{+}$ transformants were $\mathrm{Ger}^{+}$, as scored by the tetrazolium overlay procedure). None of the other three clones generated any $\mathrm{Ger}^{+}$recombinants amongst 384 transformants tested in each case. The gerC58 mutation therefore lies within the PstI-SstI region overlapping the end of gerCC and the beginning of the downstream $n d k$ gene. Since phage MY2 complements the gerC defect, however, the functional complementing component appears to be the gerCC ORF.

\section{Effects of a defect in menaquinone biosynthesis}

Attempts were made to insertionally inactivate the gerC operon, by introducing integrational plasmids $\mathrm{pHL} 1$ and pHL2 into the $B$. subtilis chromosome by transformation. Only pHL1 could be introduced, despite repeated attempts that were thoroughly controlled to ensure the competence of recipient strains. The insertion of $\mathrm{pHL} 1$ would generate a transcriptional fusion of $l a c Z$ to $n d k$, and would not result in inactivation of any gene. The failure to introduce pHL2, which would result in a disruption of the gerCC gene, suggests that gerCC is an essential gene, a conclusion consistent with the very limited viability of the gerC58 mutant, and the requirement for menaquinone in growth of $B$. subtilis (Taber, 1993).

\section{The operon designation should reflect the primary metabolic defect}

The ger $C A, \operatorname{ger} C B$ and ger $C C$ genes ought to be renamed to reflect their biosynthetic role, as proposed by KoikeTakeshita et al. (1997). However, their suggestions of heps-1, men $G$ and heps-2 for gerCA, gerCB and gerCC, respectively, should be modified, to meet the standard rules of bacterial gene nomenclature, to hepA, men $G$ and $h e p B$. The gerCC58 mutation would therefore be more correctly bepB58.

\section{Is there a relationship between menaquinone levels and spore germination?}

The bepB58 mutation must leave some residual functionality, allowing sufficient synthesis of the essential isoprenoid for the cells to grow, but very slowly, and with altered morphology. The very limited growth of the bepB58 mutant strain has precluded the measurement of menaquinone levels - it is impossible to generate a culture containing more than approximately $2 \times 10^{7}$ cells in total before suppressed derivatives take over the population. The bepB58 mutant has a more extreme defect than a strain reported to contain $<0.1 \%$ of the wild-type level of menaquinone (Farrand \& Taber, 1973).

The frequency with which the mutation can be suppressed extragenically suggests that some other function(s) in the cell may be recruited to compensate for the defect in synthesis of the heptaprenyl side chain, although these suppressed derivatives are themselves abnormal - there is a clear defect in the placing of septa in the mutants, especially in strain AM677. It is unlikely that the suppression effect is due to overexpression of the naphthoquinone ring component of menaquinone, as growth of the unsuppressed mutant was not stimulated by supplementation with the naphthoquinone menadione at levels reported to allow growth of men mutants (Leatherbarrow, 1994). It is possible that accumulation of naphthoquinone precursors might in fact be toxic to the cell, and that one class of suppressors might be down-promoter mutations in men genes. However, the nature of the suppressors is not known; it 
is even formally possible that there could be more than one suppressor in a strain, although the high frequency of occurrence of suppressed derivatives makes this less likely. Our attempts to clone the suppressors on the basis of their ability to complement the hepB58 growth defect, using gene libraries in phi105 carrying DNA from a gerC58 sup strain, yielded only clones carrying the gerC region (Leatherbarrow, 1994).

It is clear that GerC proteins are not directly concerned with the spore germination mechanism. The molecular basis of the septal abnormality and germination defect in the AM677 mutant remains to be explained, however, and it is still possible that an abnormality in menaquinone or isoprenoid biosynthesis may indirectly have an effect on membrane-associated events in germination. The morphology of the mutants suggests that processes in cell shape determination and septum formation are, again perhaps indirectly, sensitive to insufficiency of menaquinone and/or heptaprenyl isoprenoids.

\section{ACKNOWLEDGEMENTS}

This work was supported by an Iranian Government scholarship to M.A.Y. and an SERC studentship to A.J.H.L.

\section{REFERENCES}

Anagnostopoulos, C. \& Spizizen, J. (1961). Requirements for transformation in Bacillus subtilis. J Bacteriol 81, 741-746.

Farrand, S. K. \& Taber, H. W. (1973). Pleiotropic menaquinonedeficient mutant of Bacillus subtilis. J Bacteriol 115, 1021-1034.

Gollnick, P., Ishino, S., Kuroda, M. I., Henner, D. \& Yanofsky, C. (1990). The mtr locus is a two-gene operon required for transcription attenuation in the trp operon of Bacillus subtilis. Proc Natl Acad Sci USA 87, 8726-8730.

Karnovsky, M. L. (1966). A formaldehyde-glutaraldehyde fixative of high osmolality for use in electron microscopy. J Cell Biol 27, $137 \mathrm{~A}$.

Koike-Takeshita, A., Koyama, T., Obata, S. \& Ogura, K. (1995). Molecular cloning and nucleotide sequences of the genes for two essential proteins constituting a novel enzyme system for heptaprenyl diphosphate synthesis. J Biol Chem 270, 18396-18400.

Koike-Takeshita, A., Koyama, T. \& Ogura, K. (1997). Identification of a novel gene cluster participating in menaquinone (vitamin $\mathrm{K}_{2}$ ) biosynthesis. Cloning and sequence determination of the 2 heptaprenyl-1,4-naphthaquinone methyltransferase gene of Bacillus stearothermophilus. J Biol Chem 272, 12380-12383.
Leatherbarrow, A. J. H. (1994). Characterisation of the gerC and gerJ loci of Bacillus subtilis 168 . PhD thesis, University of Sheffield.

Mandel, M. \& Higa, A. (1970). Calcium-dependent phage infection. J Mol Biol 53, 159-162.

Moir, A., Lafferty, E. \& Smith, D. A. (1979). Genetic analysis of spore germination mutants of Bacillus subtilis 168 : the correlation of phenotype with map location. J Gen Microbiol 111, 165-180.

Moir, A., Yazdi, M. A. \& Kemp, E. H. (1991). Spore germination genes of Bacillus subtilis 168. Res Microbiol 142, 847-850.

Sambrook, J., Fritsch, E. F. \& Maniatis, T. (1989). Molecular Cloning: a Laboratory Manual, 2nd edn. Cold Spring Harbor, NY: Cold Spring Harbor Laboratory.

Sanger, F., Nicklen, S. \& Coulson, A. R. (1977). DNA sequencing with chain-terminating inhibitors. Proc Natl Acad Sci USA 74, 5463-5467.

Staden, R. (1990). Finding protein coding regions in genomic sequences. Methods Enzymol 183, 163-180.

Taber, H. W. (1993). Respiratory chains. In Bacillus subtilis and Other Gram-positive Bacteria: Biochemistry, Physiology, and Molecular Genetics, pp. 199-212. Edited by A. L. Sonenshein, J. A. Hoch \& R. Losick. Washington, DC: American Society for Microbiology.

Trowsdale, J. \& Smith, D. A. (1975). Isolation, characterisation and mapping of Bacillus subtilis 168 germination mutants. $J$ Bacteriol 123, 83-95.

Warburg, R. J. \& Moir, A. (1981). Properties of a mutant of Bacillus subtilis in which spore germination is blocked at a late stage. J Gen Microbiol 124, 243-253.

Yazdi, M. A. (1989). Genetic and molecular analysis of the gerC spore germination locus of Bacillus subtilis. $\mathrm{PhD}$ thesis, University of Sheffield.

Yazdi, M. A. \& Moir, A. (1990). Characterization and cloning of the gerC locus of Bacillus subtilis 168. J Gen Microbiol 136, $1335-1342$.

Zhang, Y.-W., Koyama, T. \& Ogura, K. (1997). Two cistrons of the gerC operon of Bacillus subtilis encode the two subunits of heptaprenyl diphosphate synthase. J Bacteriol 179, 1417-1419.

Zuberi, A. R., Feavers, I. M. \& Moir, A. (1985). Identification of three complementation units in the gerA spore germination locus of Bacillus subtilis. J Bacteriol 162, 756-762.

Zuberi, A. R., Ying, C., Weinreich, M. R. \& Ordal, G. (1990). Transcriptional organisation of a cloned chemotaxis locus of Bacillus subtilis. J Bacteriol 172, 1870-1876.

Received 7 January 1998; revised 31 March 1998; accepted 14 April 1998. 